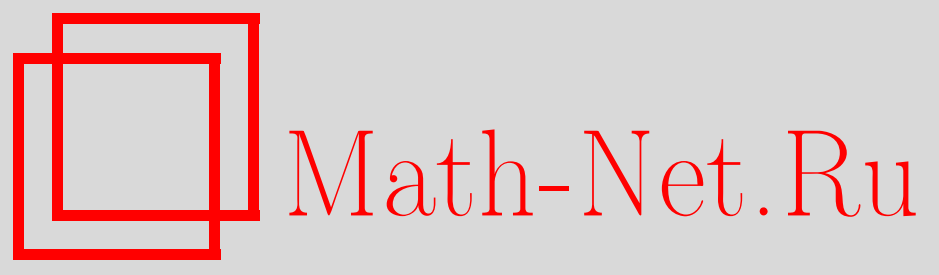

С. А. Горейнов, Н. Л. Замарашкин, Е. Е. Тыртышников, Псевдоскелетные аппроксимации при помощи подматриц наибольшего объема, Матем. заметки, 1997, том 62, выпуск 4, 619-623

DOI: https://doi.org/10.4213/mzm1644

Использование Общероссийского математического портала Math-Net.Ru подразумевает, что вы прочитали и согласны с пользовательским соглашением http://www.mathnet.ru/rus/agreement

Параметры загрузки:

IP : 52.23 .180 .231

26 апреля 2023 г., 16:03:10

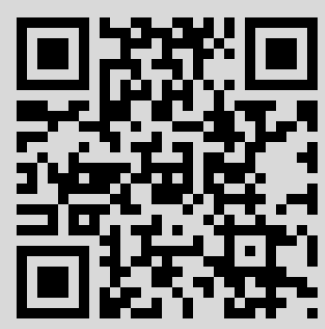




\section{ПСЕВДОСКЕЛЕТНЫЕ АППРОКСИМАЦИИ \\ ПРИ ПОМОЩИ ПОДМАТРИЦ НАИБОЛЬШЕГО ОБЪЕМА}

\section{С. А. Горейнов, Н. Л. Замарашкин, Е. Е. Тыртышников}

1. Введение. Один из подходов для быстрого умножения на плотные неструктурированные матрицы, развитый в [1], состоит в следующем. Предположим, что матрищы $A_{n}$ порождаются формулой $A_{n}=\left[f\left(x_{i}, y_{j}\right)\right]_{i, j=1}^{n}$, где $x_{i}, y_{j}$ - узлы некоторой (квазиравномерной) сетки на ограниченной области евклидова пространства $\mathbb{R}^{d}$. Тогда для класса так назьваемых асимптотически гладких функций $f$ (определение и соответствующие теоремы см. в работе [1]) оказьвается возможньм указать способ разбиения точек $x_{i}$ и $y_{j}$ нагрупшы, приводящие к разбиению матрицы на блоки малого $\varepsilon$-ранга. При этом сложность умножения на возникающий таким образом апшроксимант всей матрицы $A_{n}$ составит $O\left(n \log n \log ^{d} \varepsilon^{-1}\right)$.

Хотя практическая реализация такой идеи (связанная, например, с алгоритмами частичного сингулярного разложения) может иметь успех [2], она априори предполагает вычисление всех элементов блоков, а значит, и $O\left(n^{2}\right)$ операций. Совсем неочевидно, можно ли построить "хорошую” малоранговую аппроксимацию для блока, зная лишь небольшую часть его элементов. То, что это действительно возможно, впервые показано в [3]. Развитие соответствующей теории проведено в [4] (здесь приводится, в частности, и экспериментальное подтверждение теории для матриц, возникающих при численном решении интегрального уравнения электрического поля). Более подробно, если $\varepsilon$-ранг матрицы $A \in \mathbb{R}^{n \times n}$ не превосходит $k$, то в $A$ существуют $k$ столбцов $C \in \mathbb{R}^{n \times k}$ и $k$ строк $R \in \mathbb{R}^{k \times n}$ таких, что

$$
\|A-C G R\|_{2} \leqslant \varphi(k, n) \varepsilon
$$

где $G \in \mathbb{R}^{k \times k}$ определяется по $C$ и $R$, а функция $\varphi$ ограничена сверху полиномом малой степени.

Однако, выбор подходящих $C$ и $R$ без вычисления всех элементов $A$ представляется нам нетривиальной задачей. Теория, изложенная в [4], эту проблему не решает. Прежде всего, у нас не было до сих пор какой-либо удобной характеризации блока в пересечении нужных нам $C$ и $R$. Кажется, нам удалось, наконец, получить такую характеризацию.

Работа выполнена при поддержке Российского фонда фундаментальных исследований, грант № 97-01-00155, и фонда Volkswagen-Stiftung, грант VW I/71493.

(C) С. А. ГОРеЙнов, Н. Л. ЗАмАРАшкин, Е. Е. Тыртышников 
В настоящей работе мы доказываем оценки типа (1) для строк и столбцов, дающих в пересечении подматрицу со следующим экстремальным свойством: среди всех подматриц матрицы $A$ размера $k \times k$ она имеет максимальный по модулю детерминант. Другими словами, чтобы получить “хорошие” строки и столбцы, достаточно выбрать подматрицу наибольшего “объема" (или отличающуюся от таковой не очень сильно). Заметим, что подматрицы наибольшего объема возникают и оказьваются весьма полезными в некоторых классических задачах теории аппроксимации функций (см., например, [5]).

Успех практической реализации нашего подхода определяется тем, насколько эффективно мы умеем находить подматрищы наибольшего объема. Очевидно, чтобы сделать это, не имея всех элементов матрицы, необходима некоторая априорная информация о ее происхождении. Если матрица является дискретным аналогом некоторого (интегрального) оператора, то такая информация обычно имеется.

2. Обозначения, определения и предварительные замечания. Для любой квадратной матрицы $A \in \mathbb{R}^{n \times n}$ введем ее $\tau$-nсевдообратную $A_{\tau}^{\dagger}$ в соответствии с сингулярным разложением $A$ :

$$
A=U_{A} \Sigma_{A} V_{A}, \quad \Sigma_{A}=\operatorname{diag}\left(\sigma_{A 1}, \ldots, \sigma_{A n}\right), \quad U_{A}^{T} U_{A}=V_{A} V_{A}^{T}=I,
$$

по следующей формуле:

$$
A_{\tau}^{\dagger}=V_{A}^{T} \Sigma_{A}^{\dagger} U_{A}^{T}, \quad \text { где } \Sigma_{A}^{\dagger}=\operatorname{diag}\left(\sigma_{A 1}^{\dagger}, \ldots, \sigma_{A n}^{\dagger}\right), \quad \sigma_{A i}^{\dagger}= \begin{cases}\sigma_{A i}^{-1}, & \sigma_{A i} \geqslant \tau \\ 0, & \sigma_{A i}<\tau\end{cases}
$$

Обгемом $v(A)$ квадратной матрищы $A$ будем назьвать модуль ее детерминанта. Сингулярные числа всякой матрицы $A$ будем упорядочивать по убыванию так, что $\sigma_{1}(A) \geqslant$ $\sigma_{2}(A) \geqslant \cdots \geqslant \sigma_{n}(A)$. Малоранговую аппроксимацию матрицы $A$ вида $C G R$, где $R$ и $C$ - некоторые строки и столбцы $A$, которые в пересечении дают опорную подматрицу матрицы $A$, будем назьвать псевдоскелетной компонентой $A$ (см. [4]). Если $M-$ невырожденная опорная подматрища, то, как будет показано ниже, оценки типа (1) получаются при выборе $G=M^{-1}$. С практической точки зрения важно то, что в случае вырожденной или плохо обусловленной опорной подматрицы можно взять $G=M_{\tau}^{\dagger}$ при некотором $\tau>0$. При этом погрешность аппроксимации изменится таким образом, как будто $\varepsilon$ увеличилось на $\delta$.

Нас будет интересовать погрешность псевдоскелетной аппроксимации, опирающейся на $k \times k$-подматрищу максимального объема в матрище $A$ такой, что

$$
A \in \mathbb{C}^{n \times n}, \quad Z=A-F, \quad \operatorname{rank} Z=k, \quad\|F\|_{2} \leqslant \varepsilon .
$$

Пусть $v_{\max }^{(k)}(X)$ обозначает максимально возможньй объем подматрицы порядка $k$ в матрице $X$. Символом $c$ будем обозначать любую положительную константу (возможно, каждьй раз разную).

Нам понадобятся классические неравенства разделения в следующей форме.

Лемма. Пусть $B=\left[\begin{array}{ll}B_{1} & b_{2}\end{array}\right] \in \mathbb{C}^{m \times n}, b_{2} \in \mathbb{C}^{m \times 1}$. Тогда $\sigma_{1}(B) \geqslant \sigma_{1}\left(B_{1}\right) \geqslant$ $\sigma_{2}\left(B_{2}\right) \geqslant \cdots \geqslant \sigma_{p-1}\left(B_{1}\right) \geqslant \sigma_{p}(B)$, где $p=\min (m, n)$. 


\section{3. Основные результаты.}

ТЕОРема 1. Пусть матрица А, рассматриваемая в блочном разбиении

$$
A=\left[\begin{array}{ll}
a_{11} & a_{12} \\
a_{21} & a_{22}
\end{array}\right] \in \mathbb{C}^{n \times n}, \quad a_{22} \in \mathbb{C}^{k \times k},
$$

такова, что $\sigma_{k+1}(A) \leqslant \varepsilon$ и, кроме того, $a_{22}$ невырождена. Тогда

$$
\left\|A-\left[\begin{array}{l}
a_{12} \\
a_{22}
\end{array}\right] a_{22}^{-1}\left[\begin{array}{ll}
a_{21} & a_{22}
\end{array}\right]\right\|_{2} \leqslant c \varepsilon\left(1+\left\|a_{12} a_{22}^{-1}\right\|_{2}^{2}\right)^{1 / 2} .
$$

ТЕОремА 2. Если, дополнительно, $v\left(a_{22}\right) \geqslant \gamma v_{\max }^{(k)}(A)$, mo

$$
\left\|A-\left[\begin{array}{l}
a_{12} \\
a_{22}
\end{array}\right] a_{22}^{-1}\left[\begin{array}{ll}
a_{21} & a_{22}
\end{array}\right]\right\|_{2} \leqslant c \varepsilon \sqrt{1+k(n-k) \gamma^{-2}} .
$$

ДокАЗАТЕЛЬСТво будет опираться на невырожденность $A$, что мы можем без ограничения обшности потребовать, поскольку иначе матрица

$$
\widetilde{A}=\left[\begin{array}{cc}
a_{11}+\delta I & a_{12} \\
a_{21} & a_{22}
\end{array}\right]
$$

1) невырождена для всех достаточно мальх $\delta$;

2) близка к $A$ : при $\delta \leqslant \varepsilon\|\widetilde{A}-A\|_{2} \leqslant \varepsilon \Longrightarrow \sigma_{k+1}(\widetilde{A}) \leqslant 2 \varepsilon$;

$3)$ совпадает с $A$ во втором блочном столбце, а значит, блок $(2,2)$ обеспечивает ту же оценку в правой части (3).

Поэтому мы будем строить псевдоскелетные аппроксимации для $\widetilde{A}$; поскольку в получаемой оценке константа $c$ обозначает некоторое положительное число, такая же оценка будет справедлива и для $A$.

Пусть теперь $A$ невырождена. Запишем $A^{-1}$ в блочном разбиении, совпадающем с указанным в (2):

$$
A^{-1}=\left[\begin{array}{ll}
b_{11} & b_{12} \\
b_{21} & b_{22}
\end{array}\right] .
$$

В силу формул $\Phi$ робениуса $b_{11}=\left(a_{11}-a_{12} a_{22}^{-1} a_{21}\right)^{-1}$; таким образом, блок $b_{11}$ напрямую связан с погрешностью аппроксимации

$$
\sigma_{1}\left(b_{11}^{-1}\right)=\left\|A-\left[\begin{array}{l}
a_{12} \\
a_{22}
\end{array}\right] a_{22}^{-1}\left[\begin{array}{ll}
a_{21} & a_{22}
\end{array}\right]\right\|_{2} .
$$

Поэтому запишем еще одну формулу, содержащую $b_{11}$ :

$$
b_{11}^{-1}\left[\begin{array}{ll}
b_{11} & b_{12}
\end{array}\right]=\left[\begin{array}{ll}
I & -a_{12} a_{22}^{-1}
\end{array}\right] .
$$

Она легко следует из тождества $A^{-1} A=I$, или точнее $b_{11} a_{12}+b_{12} a_{22}=0$. 
Понятно, что $\sigma_{n-k}\left(\left[\begin{array}{ll}b_{11} & b_{12}\end{array}\right]\right)>0$. Верно даже большее:

$$
\sigma_{k+1}(A) \leqslant \varepsilon \Longleftrightarrow \sigma_{n-k}\left(A^{-1}\right) \geqslant \varepsilon^{-1} \Longrightarrow \sigma_{n-k}\left(\left[\begin{array}{ll}
b_{11} & b_{12}
\end{array}\right]\right) \geqslant \varepsilon^{-1},
$$

где последний переход осуществляется $k$-кратным применением леммы. Но тогда справедливо следующее неравенство, легко вытекающее из вариационных формул для сингулярных чисел:

$$
\sigma_{1}\left(b_{11}^{-1}\left[\begin{array}{ll}
b_{11} & b_{12}
\end{array}\right]\right) \geqslant \sigma_{1}\left(b_{11}^{-1}\right) \sigma_{n-k}\left(\left[\begin{array}{ll}
b_{11} & b_{12}
\end{array}\right]\right) \geqslant \sigma_{1}\left(b_{11}^{-1}\right) \varepsilon^{-1} .
$$

Собирая вместе (4)-(6), получаем

$$
\left\|A-\left[\begin{array}{l}
a_{12} \\
a_{22}
\end{array}\right] a_{22}^{-1}\left[\begin{array}{ll}
a_{21} & a_{22}
\end{array}\right]\right\|_{2} \leqslant \varepsilon\left(1+\left\|a_{12} a_{22}^{-1}\right\|_{2}^{2}\right)^{1 / 2} .
$$

ДокАЗАТЕЛЬСТВО теоремы 2 есть вариация основной леммы из работы [4], и, тем не менее, мы его приведем.

Рассмотрим тождество

$$
\left[\begin{array}{l}
a_{12} \\
a_{22}
\end{array}\right] a_{22}^{-1}=\left[\begin{array}{c}
a_{12} a_{22}^{-1} \\
I
\end{array}\right]
$$

Для удобства обозначим матрищы в квадратных скобках символами $A_{2}$ и $C_{2}$, так что $A_{2} a_{22}^{-1}=C_{2}$. Подматрица наибольшего объема сосредоточена в одних и тех же строках $A_{2}$ и $C_{2}$ в силу невырожденности $a_{22}$. Более того, если

$$
v(a) \geqslant \gamma v_{\max }\left(A_{2}\right)
$$

где $a$ - некоторая подматрица $A_{2}$, то

$$
v(c)=v(a) v^{-1}\left(a_{22}\right) \geqslant \gamma v_{\max }\left(A_{2}\right) v^{-1}\left(a_{22}\right)=\gamma v_{\max }\left(C_{2}\right),
$$

где $c$ - такая же подматрица $C_{2}$.

Предположим теперь, что какой-то элемент матрицы $a_{12} a_{22}^{-1}$ по модулю превьшает $\gamma^{-1}$. Переставив две строки в $C_{2}$, мы можем добиться того, что этот элемент займет место одной из единищ в нижнем блоке $C_{2}$. Это всего лишш означает, что мы увеличили объем нижнего блока более чем в $\gamma^{-1}$ раз, или в силу $(7),(8)$ что для какой-то $а$ будет $v(a)>\gamma^{-1} v\left(a_{22}\right)$, что невозможно: $v\left(a_{22}\right) \geqslant \gamma v_{\max }\left(A_{2}\right)$.

Таким образом, все элементы $a_{12} a_{22}^{-1} \in \mathbb{C}^{(n-k) \times k}$ ограничены по модулю, откуда $\left\|a_{12} a_{22}^{-1}\right\|_{2}^{2} \leqslant k(n-k) \gamma^{-2}$.

3. Оценка в случае $\tau$-псевдообращения опорной подматрицы. Как видно из формулировки теоремы 1 , аппроксимация на подматрице $a_{22}$ не зависит от того, насколько близко младшее сингулярное число $a_{22} \mathrm{k} \varepsilon$. Однако, матрища $a_{22}^{-1}$, на самом деле, зависит от этого довольно сильно, особенно если $\varepsilon$ мало. Актуальность ситуации связана с тем, что мы, как правило, располагаем лишш оценкой сверху на нужный нам $\varepsilon$-ранг. 
ТЕОрема 3. В условиях теоремы 1 имеем оченку

$$
\left\|A-\left[\begin{array}{l}
a_{12} \\
a_{22}
\end{array}\right] a_{22}^{\dagger}\left[\begin{array}{ll}
a_{21} & a_{22}
\end{array}\right]\right\|_{2} \leqslant c(\varepsilon+\tau)\left(1+s^{2}\right)^{1 / 2},
$$

где $s=\max \left\{\left\|a_{22}^{-1} a_{21}\right\|_{2},\left\|a_{12} a_{22}^{-1}\right\|_{2}\right\}$.

ДоКАЗАТЕльСтво. Как и прежде, оценим нормы блоков матришы погрешности, которая на этот раз имеет вид

$$
\left[\begin{array}{ll}
a_{11}-a_{12} a_{22}^{\dagger} a_{21} & a_{12}-a_{12} a_{22} \underset{\tau}{\dagger} a_{22} \\
a_{21}-a_{22} a_{22} a_{21} & a_{22}-a_{22} a_{22} a_{22}
\end{array}\right] .
$$

Проще всего это сделать для блока $(2,2)$; очевидно, $\left\|\left(I-a_{22} a_{22}^{\dagger}\right) a_{22}\right\|_{2} \leqslant \tau$. Далее,

$$
\left\|a_{21}-a_{22} a_{22}^{\dagger} a_{21}^{\dagger}\right\|_{2} \leqslant\left\|\left(I-a_{22} a_{22}^{\dagger}\right) a_{22}^{-1} a_{22} a_{21}\right\|_{2} \leqslant \tau\left\|a_{22}^{-1} a_{21}\right\|_{2},
$$

и аналогично для блока $(1,2)$; отметим, что мы используем невырожденность $a_{22}$. Чтобы получить оценку для блока $(1,1)$, рассмотрим матрицу

$$
\widetilde{A}=\left[\begin{array}{cc}
a_{11} & a_{12} \\
a_{22} a_{22}^{\dagger} a_{21} & a_{22}
\end{array}\right], \quad \text { для которой }\left\|\widetilde{A}-\left[\begin{array}{ll}
I & \\
& a_{22} a_{22}^{\dagger}
\end{array}\right] A\right\|_{2} \leqslant \tau,
$$

откуда в силу оценок типа $\sigma_{i}(A+E) \leqslant \sigma_{i}(A)+\sigma_{i}(E)$ и $\sigma_{i}(A B) \leqslant \sigma_{i}(A) \sigma_{i}(B)$

$$
\sigma_{k+1}(\widetilde{A}) \leqslant \tau+\sigma_{k+1}\left(\left[\begin{array}{ll}
I & \\
& a_{22} a_{22}^{\dagger}
\end{array}\right] A\right) \leqslant \tau+\sigma_{k+1}(A) \leqslant \tau+\varepsilon .
$$

Применяя теорему 1 к матрице $\widetilde{A}$, получаем

$$
\left\|a_{11}-a_{12} a_{22}^{-1} a_{22} a_{22}{ }_{\tau}^{\dagger} a_{21}\right\|_{2} \leqslant c(\tau+\varepsilon)\left(1+\left\|a_{12} a_{22}^{-1}\right\|_{2}^{2}\right)^{1 / 2} .
$$

Осталось воспользоваться соотношением

$$
\|A\|_{2}^{2} \leqslant \sum_{i, j}\left\|A_{i j}\right\|_{2}^{2}
$$

\section{СПИСОК ЦИТИРОВАННОЙ ЛИТЕРАТУРЫ}

1. Tyrtyshnikov E. E. // Numerical Analysis and Its Applications. Proceedings of WNAA-96. Lecture Notes in Comput. Sci. V. 1196. New York: Springer Verlag, 1996. P. 505-516. 2. Goreinov S.A., Tyrtyshnikov E. E., Yeremin A. Yu. Matrix-free iterative solution strategies for large dense linear systems // Numer. Linear Algebra Appl. (to appear). 3. Горейнов С. A., Замарашкин Н. Л., Тыртышников Е. Е. // Докл. РАН. 1995. Т. 343. № 2. С. 151-152. 4. Goreinov S. A., Tyrtyshnikov E. E., Zamarashkin N. L. A theory of pseudo-skeleton approximations // Linear Algebra Appl. (to appear). 5. Гайер Д. Лекции по теории аппроксимации в комплексной плоскости. М.: Мир, 1986. 